\title{
CALPHAD-Based Methods for Alloy Additive Manufacturing
}

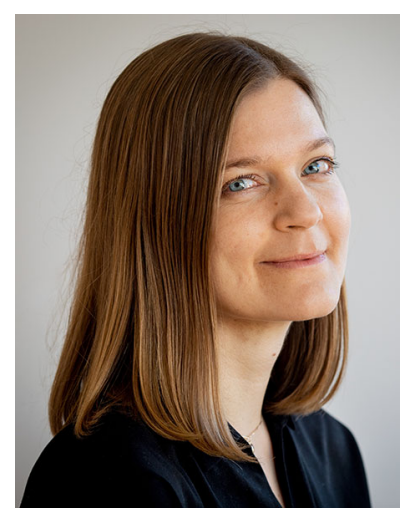

Greta Lindwall

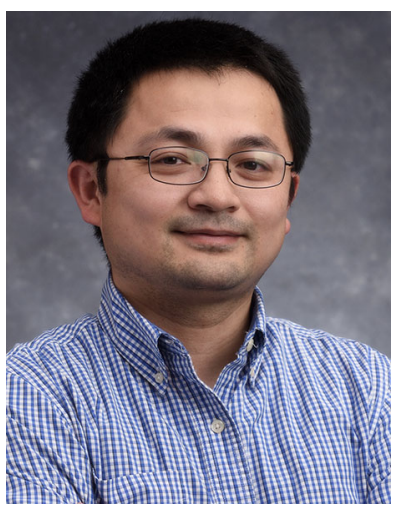

Wei Xiong
Additive manufacturing (AM) or 3D printing has shown great potential for building complex structures using layerby-layer deposition and enhancing materials performance through dissimilar materials processing. In addition to enlarging the design space for processing new components with hierarchical structures, the development of the AM techniques opens the door to new alloy designs and postprocessing routes. For this, the knowledge of stable and metastable phase equilibria and reaction pathways is essential.

Among computational methods, the CALPHAD (CALculation of PHAse Diagrams) method has a unique position to support the AM technology development by providing multicomponent and multiphase descriptions enabling the coupling of mechanical and material models. Moreover, CALPHAD can effectively be integrated with other computational and experimental methods to better understand the complex process-structure-property relationships in AM. The post-AM process is critical in AM design, and the CALPHAD method can assist post-heat treatment optimization in adapting existing castable or weldable alloys for AM.

For example, CALPHAD-based computational tools can be used to elucidate process-structure relationships by predictions of property diagrams, micro-segregation during solidification, diffusion-controlled phase transformation and precipitation, as well as diffusionless phase transformations. Thus, CALPHAD offers many sophisticated models to capture the physics of non-equilibria in multicomponent systems.

However, what are the current limitations of CALPHAD in terms of reliability when performing predictions for metal AM? How can the CALPHAD-based models be further developed to enable efficient alloy design for AM? And looking forward, machine learning has demonstrated its capability to further enhance traditional modeling approaches. Since the machine learning models require reliable databases, the importance of the physics-based 
model and the materials databases based on the CALPHAD method should not be underestimated.

The collection of articles on AM in this issue shows case studies on the integration of experiments and CALPHADbased modeling approaches. It is expected that these studies will be stimulating to the community and thus inspire more effective modeling and experimental efforts for AM design.

\section{Greta Lindwall}

Guest Editor

Journal of Phase Equilibria and Diffusion

KTH Royal Institute of Technology

Stockholm, Sweden

Contact e-mail: gretal@kth.se

\section{Wei Xiong}

Guest Editor

Journal of Phase Equilibria and Diffusion

University of Pittsburgh

Pittsburgh, Pennsylvania, USA

Publisher's Note Springer Nature remains neutral with regard to jurisdictional claims in published maps and institutional affiliations. 